\title{
Retraction Note to: X-ray observations of PSR B0335+54 and its pulsar wind nebula
}

Katherine E. McGowan, W. Thomas Vestrand, Jamie A. Kennea, Silvia Zane, Mark Cropper and France A. Córdova

\section{Retraction Note to:}

Chapter "X-ray observations of PSR B0335+54 and its pulsar wind nebula" in: S. Zane, R. Turolla and D. Page (eds), Isolated Neutron Stars: From the Surface to the Interior, Springer https://doi.org/10.1007/978-1-4020-5998-8_39

The authors have retracted this chapter [1]. This work had been unintentionally submitted and published in full length as part of a conference proceedings [2]. However, the authors had already published the content in another journal [3] and have now retracted [4] the article in the conference proceedings [2]. This chapter is therefore also redundant. All authors agree with this retraction.

[1] McGowan K.E., Vestrand W.T., Kennea J., Zane S., Cropper M., Córdova F.A. (2007) X-ray observations of PSR B0355+54 and its pulsar wind nebula. In: Zane S., Turolla R., Page D. (eds) Isolated Neutron Stars: From the Surface to the Interior. Springer, Dordrecht https://doi.org/10.1007/978-1-4020-5998-8_39

[2] Retracted article: McGowan, K.E., Vestrand, W.T., Kennea, J.A. et al. Astrophys Space Sci (2007) 308: 309. https://doi.org/10.1007/s10509-007-9319-9

[3] McGowan, K.E., Vestrand, W.T., Kennea, J.A. et al. Astrophys J (2006) 647: 1300. https://doi.org/10.1086/505522

[4] McGowan, K.E., Vestrand, W.T., Kennea, J.A. et al. Astrophys Space Sci (2019) 364: 128. https://doi.org/10.1007/s10509-019-3622-0

The retracted version of this chapter can be found at https://doi.org/10.1007/978-1-4020-5998-8_39

(C) Springer Science+Business Media B.V., 2019

S. Zane et al. (eds.), Isolated Neutron Starts: From the Surface to the Interior, https://doi.org/10.1007/978-1-4020-5998-8_91 